\title{
Monitoring of the degradation dynamics of agricultural films by IR thermography
}

\author{
by P. Mormile ${ }^{*}$, L. Petti ${ }^{*}$, M. Rippa ${ }^{*}$, B. Immirzi ${ }^{* \star}$, M. Malinconico ${ }^{* \star}$ \\ * Istituto di Cibernetica-CNR, via Campi Flegrei 34, 80078 Pozzuoli \\ ** Istituto di Chimica e Tecnologia dei polimeri-CNR, via Campi Flegrei 34, \\ 80078 Pozzuoli
}

\begin{abstract}
The high consumption of plastic materials for use in agriculture is generating serious problems regarding environmental impact. Biodegradable materials are at present the only solution to this type of problems. The research in Material Science is proposing day by day different materials for application in various sectors. One of the problems in this activity is to analyse the degradation states of the films before their application in order to know the time and the modality of degradation. Here we propose a novel method based on thermography vs time which allows to evaluate the degradation dynamics of agricultural films.
\end{abstract}

\section{Introduction}

The consumptions of plastic materials for application in agriculture are dramatically increasing in the last decades posing serious problems of environmental concerns. As shown in Fig. 1, the trend is positive for each type of plastic product used in agriculture.

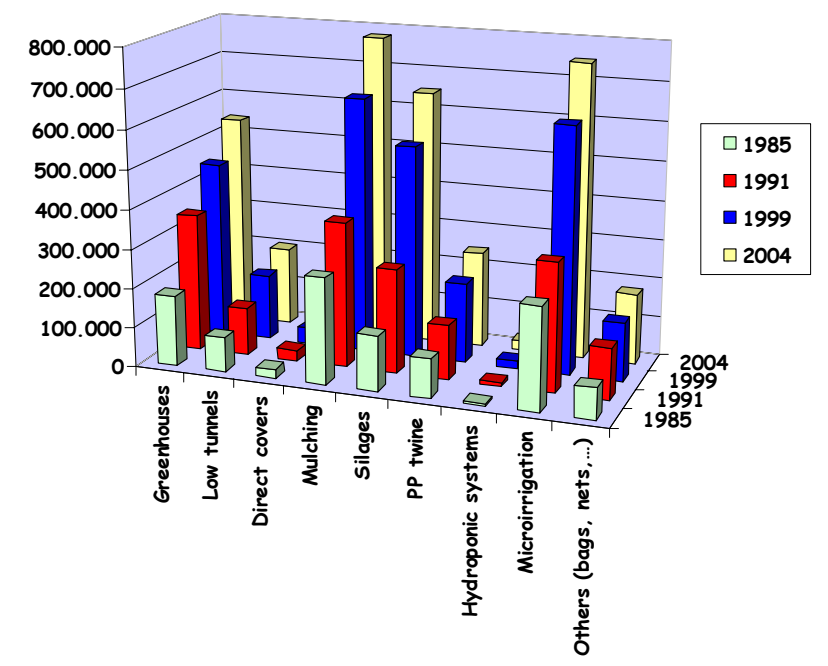

Fig. 1: Quantities distribution (Tons) of plastic products used in agriculture in the last two decades. 
On the other hand, the enormous consumption of plastic films, gives rise to plastic waste which is increasing more and more. In Table 1 we report the incidence (\%) on the total plastic waste for each item.

$\begin{array}{lll}\text { Sources: } & \text { household } & 63.0 \% \\ & \text { packaging } & 15.0 \% \\ & \text { automotive } & 5.5 \% \\ & \text { building } & 5.0 \% \\ \text { electric/electronic } & 5.0 \% \\ & \text { agriculture } & 2.0 \%\end{array}$

Table 1. Reported values in percentages of plastic waste in different application fields.

Even if the percentage of plastic waste in agriculture seems quite low compared to the other uses, it represents the main danger to the environment due to the difficulties in entering into the recycling chain. In other words, the landfill of million and million tons of plastic films represents a serious problem for the environment also because, while consumption continuously increases, the recycling system in any country doesn't work as it should. Practically all these films are collected by the farmers and left on the ground borders to be incinerated. The same collection by the farmer is difficult; these films being thinner and damaged, their removal through automatic means is almost impossible.

Biodegradable materials are the ideal solution to this kind of problems which are involving the whole channel food year by year.

In this scenery the effort of the research world, including materials engineers, chemists, physicists, and agronomists, focused on new materials with biodegradable properties to be employed in agriculture.

The research, in this frame, requires new investigation tools in order to evaluate the real biodegradability of new materials, the degradation times together with its dynamics, and the performances when they are applied on soil. In this view, our recent activity is focused on looking for innovative methodologies. Here we report the first experimental evidence of a novel method which uses IR thermography to monitor the degradation states of the proposed materials.

\section{Materials and Methods}

In the last three years, within a European "LIFE-Environment" Project funded by EC and entitled "Biodegradable coverages for sustainable agriculture (BIOCOAGRI)", we devoted our research activity, together with other partners, to prepare, characterise, study and analyse new type of materials with biodegradable properties useful for agriculture uses (mulch and solarization).

In the Life-project it was aimed at sprayable mulching and solarisation films. The films should be biodegradable, non-toxic and originate from renewable resources and therefore polysaccharides were chosen to be the basic ingredients in the waterborne formulations developed. In the recent years starch-based materials have been proposed to produce agriculture film for mulching. The limits of this type of products are the degradation times and the cost very high. Recently, the research has focused on an innovative approach where a sort of sprayable water-based varnish is applied on soil. Such varnish, made of biodegradable polysaccharides mixture, contains biodegradable plasticizers to allow the film to remain elastic for the time needed. Moreover, a set of fibrous natural fillers have been tested, which can be mixed in the varnish or can be preventively applied on soil. 
We considered several materials with different origin and performed many measurements in order to test and verify their chemical and physical properties.

Among these materials we focused our interest on three type of polysaccharides materials with the best appealing properties:

PSS - This formulation consists of a mixture of the galactomannans Locust bean gum and Guar gum, in water, to which has been added a non-gelling concentration of agarose. This formulation dries into a clear film and stable towards rain.

Chitosan (from crustaceous) acts as a preservative coating and biofungicide when sprayed on plants and vegetables. Plant seeds are soaked in a very low concentrations of chitosan aqueous solutions to both prevent microbial infections and increase the plant production. Chitosan, in fact, can trigger defensive mechanism in plants against infections and parasite attacks.

Alginates - The Alginate films are based on a polysaccharide, the Sodium Alginate obtained from brown seeweed. The polysaccharide used is of course biodegradable and soluble in water; in presence of divalent cations, such as calcium, it gives rise to threedimensional, thermo irreversible, stable and insoluble network (gel). In particular the Sodium Alginate used comes from China and by NMR analysis, it results rich in guluronic acid, responsible of the rapid cross-linking in presence of calcium; on the base of this important property, it has been prepared water solutions based on Chinese sodium alginate and sprayed them on the soil; the calcium naturally present in the soil should assure the cross-linking of the polymer, allowing its permanence for the lifetime compatible with the agricultural cultivation.

In table 2, we report some of the considered materials and some measured characteristics. The radiometric tests show that the sprayable formulations exhibit a good capacity to reduce weeds growth in the soil and in the substrate, due to their low PAR (Photo Active Region) transmissivity: at the same time they have a high capacity of storing energy in the soil due to their low transmissivity in the LWIR (long wave infrared) range. In the following table we report a summary of the functional properties of the films according to the radiometric tests planned for the project.

\begin{tabular}{|l|c|c|c|}
\hline \multicolumn{1}{|c|}{ Film } & $\begin{array}{c}\text { Light Transmission } \\
\text { in PAR (\%) } \\
(400-700 \mathrm{~nm})\end{array}$ & $\begin{array}{c}\text { Light Transmission } \\
\text { in NIR (\%) } \\
(700-1200 \mathrm{~nm})\end{array}$ & $\begin{array}{c}\text { Thermicity } \\
(\%)\end{array}$ \\
\hline PSS Spray & 91 & 90 & 98 \\
\hline Alginate/Starch C./Glycerol & 96 & 95 & 97 \\
\hline Alginate/PolyGlycerol & 3,3 & 69 & 94 \\
\hline Chitosan\PolyGlycerol & 94 & 95 & 98 \\
\hline \begin{tabular}{l} 
ChitosanIPolyGlycerol \} $\\
{\text { Carbon Black }}$ & 1,7 & 1,5 & - \\
\hline
\end{tabular}
\end{tabular}

Table 2. Averaged values of light transmission in the ultraviolet-visible - near infrared regions and an estimation of thermicity value.

The agronomic performances have been measured, together with mechanical properties and degradation behavior. The results seem to confirm the technical feasibility of such approach in developing a sustainable plasticulture.

One of the activities planned for the project was the acquisition of information about the degradation process of the films. Normally in order to study this process several pictures are taken at different times and then analysed. The comparison between the photos of the first week, of the second one and so on, indicate that the film is modifying itself. The 
novelty that we introduced is the use of thermography to monitor the dynamic of the degradation process. This approach allows to evaluate the local modifications of the material investigated as a function of time and according to the changing of the thermal properties induced by the degradation dynamics.

In our experiment we put on soil in open fields some of the materials of interest. The main characteristic of these materials is that they are originally liquid and they are sprayed on soil and immediately after they cross-link in a film state. This is the first time that sprayable films are proposed in agriculture for mulching soil instead of the traditional plastic ones.

The IR thermal camera which we employed (see Figure 3) is an FTI6 supplied by Land Co. which provides accurate and highly stable industrial radiometric imaging and temperature measurement from -20 to $2000^{\circ} \mathrm{C}$ with a spectral response ranging from 3.2 to $4.2 \mu \mathrm{m}$ and a display resolution of $0.1^{\circ} \mathrm{C}$.

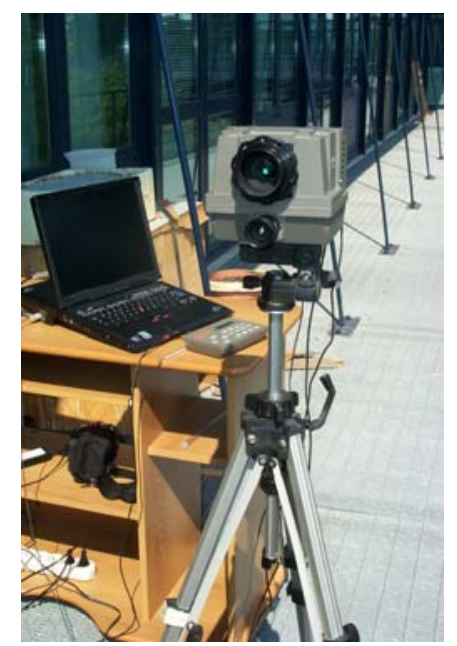

Fig. 3. Thermography station
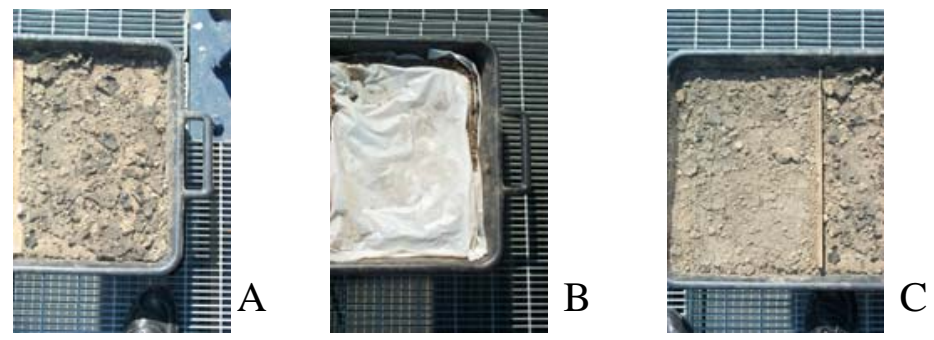

Fig. 4. Materials under study;

A: PSS Spray; B: Biodegradable commercialised film; C: Alginate based Spray.

\section{Results and discussion}

Through an IR thermal camera we monitored the state of the films day by day for two weeks, as reported in figure 4 , and we analysed the thermal images to detect the 
continuous changing of them. Thanks to the dedicated software we studied also the temperature profiles in a selected target area of each material.

In figures 5-6 we report the images after an elaboration describing the dynamic process of the degradation of a PSS spray and of the alginate-based spray, respectively.

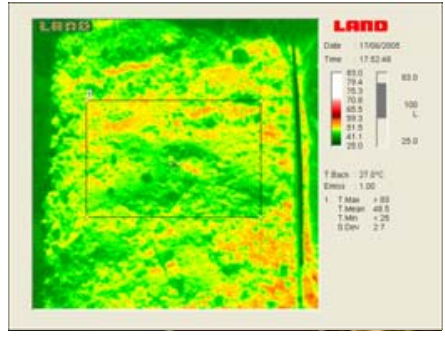

Bare soil

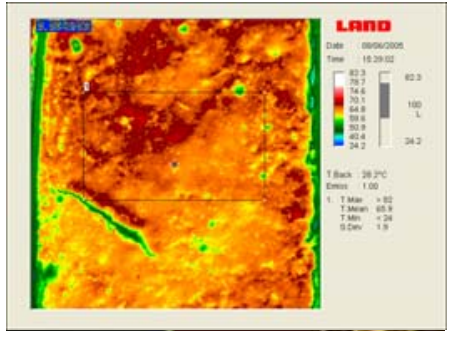

Film at the starting trial

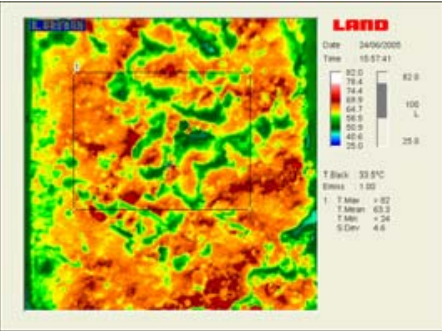

Degradated film after two weeks

Fig. 5. Thermal images of a PSS Spray analysed vs time

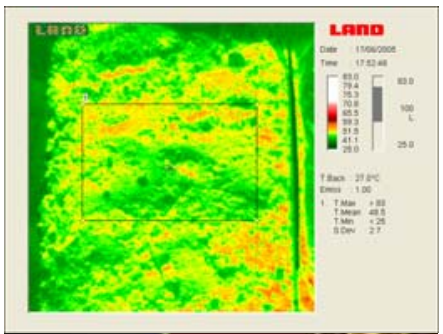

Bare soil

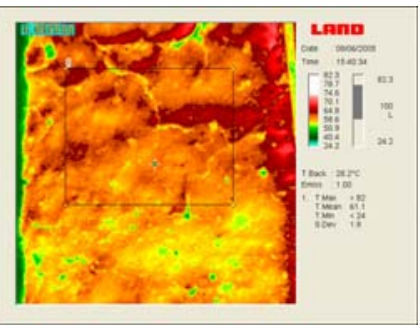

Film at the starting trial

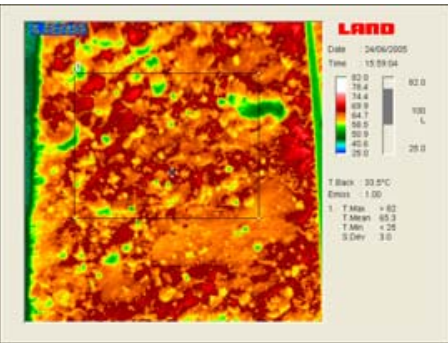

Degradated film after two weeks

Fig. 6. Thermal images of an Alginate-based Spray analysed vs time 
In figure 7, it is reported the ratio of the average temperature per unit area of the films on the soil temperature as a function of time. This ratio as shown in figure decreases according to the degradation dynamic. It is worthwhile to notice that the values of two different sprayable biodegradable materials (c-d) after two weeks come to converge with the value of bare soil (b) and of a commercialised biodegradable film (a).

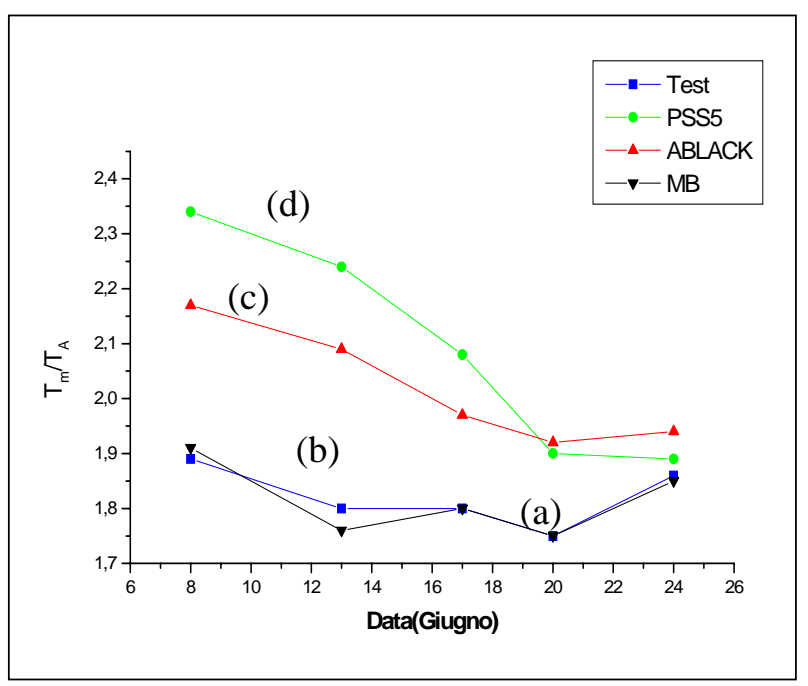

Fig. 7. Experimental data reporting the normalised average temperatures per unit area of each material vs time; (a): biodegradable commercialised film; (b): bare soil;

(c), (d) biodegradable sprayed films.

The new methodological approach introduced by us in this paper, represents a valid alternative to the traditional ones, based on the visual control at different weeks. While the analysis in the visible region gives information on the effect of the "phenomenon degradation", the monitoring day by day through IR thermography extends the study to the causes of the degradation. As well known, the degradation of a film is affected by many factors ranging from different species of bacteria in soil to the chemical nature of the soil, from the local climate conditions to the chemical agents used by the farmer. Thanks to our method, it is possible to correlate these factors and to evaluate the role of each components and its contribute to the degradation state. Furthermore, it's possible to monitor the dynamic of degradation acquiring information before the tearing of the film. As it is shown in figure 7, in correspondence of a modification of the physical and chemical structure of the film, its emittivity changes giving rise to an higher heat transmission which is detected in the tearing area. This behaviour is confirmed by the data reported in figure 8 . The bigger is the tearing (degradation state) the higher is the temperature detected. 
http://dx.doi.org/10.21611/qirt.2006.052

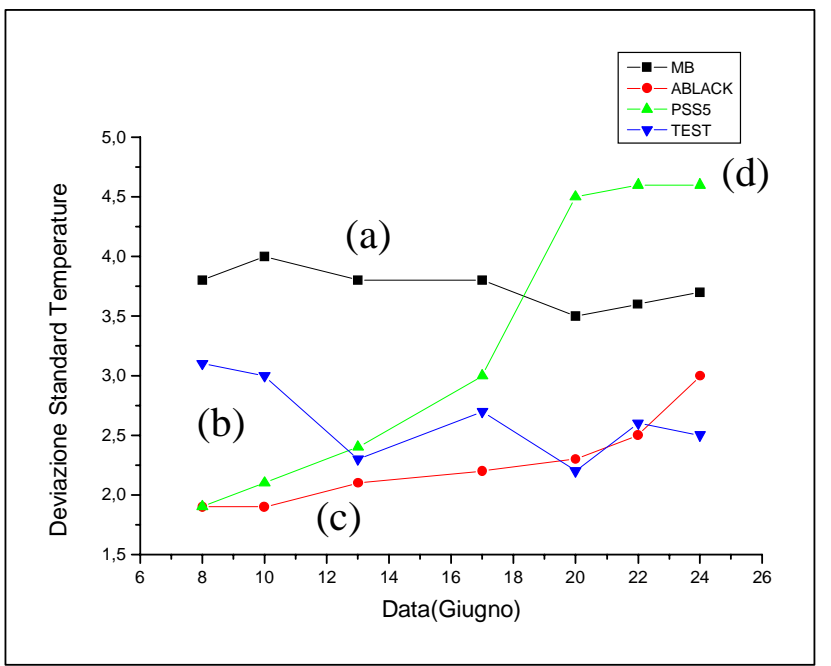

Fig. 8. Experimental data reporting the standard deviation of the temperatures per unit area of each material vs time; (a): biodegradable commercialised film; (b): bare soil; (c), (d) biodegradable sprayed films.

In conclusion, our experimental results show that thermography technique can be a very good tool in order to monitor the degradation process of a film in the agriculture field but also to give information on the degradation mechanism related to the soil state. Furthermore we believe that the ratio values of temperatures reported in figure 7 could be considered as a reference parameter in order to indicate the degradation state of a material employed in agriculture.

\section{Acknowledgments}

This work has been partially supported by the European Life Project Bio.Co.AGRI contract 03/377. 